\title{
Assembly of Hydrogen Bonded Diamondoid networks Based on Synthetic Metal-Organic Tetrahedral Nodes
}

Bao-Qing Ma, ${ }^{*}$ Hao-Ling Sun and Song Gao*

\section{Supporting materials}

\section{SHG setups:}

SGH experiment: the experimental set up included a high-power mode-locked Nd-YAG laser system with a selected wavelength of $1064 \mathrm{~nm}$ as incident beam. The laser beam is split into two parts: one to generate the second harmonic signal in the sample and the other to generate the second harmonic signal in the reference KDP powder. 

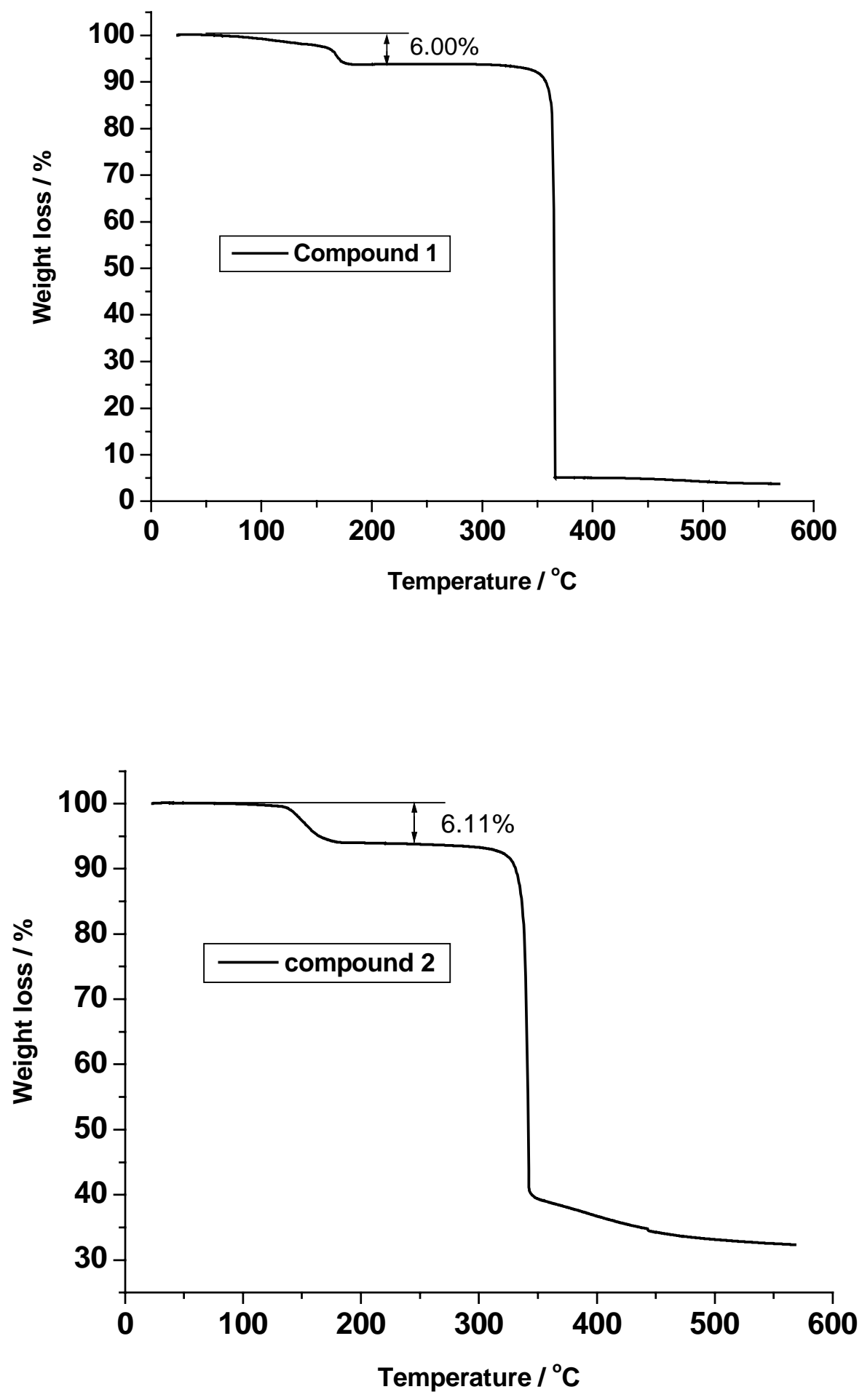

Fig. S1. Thermogravimetric Curve for compounds 1 and 2. 

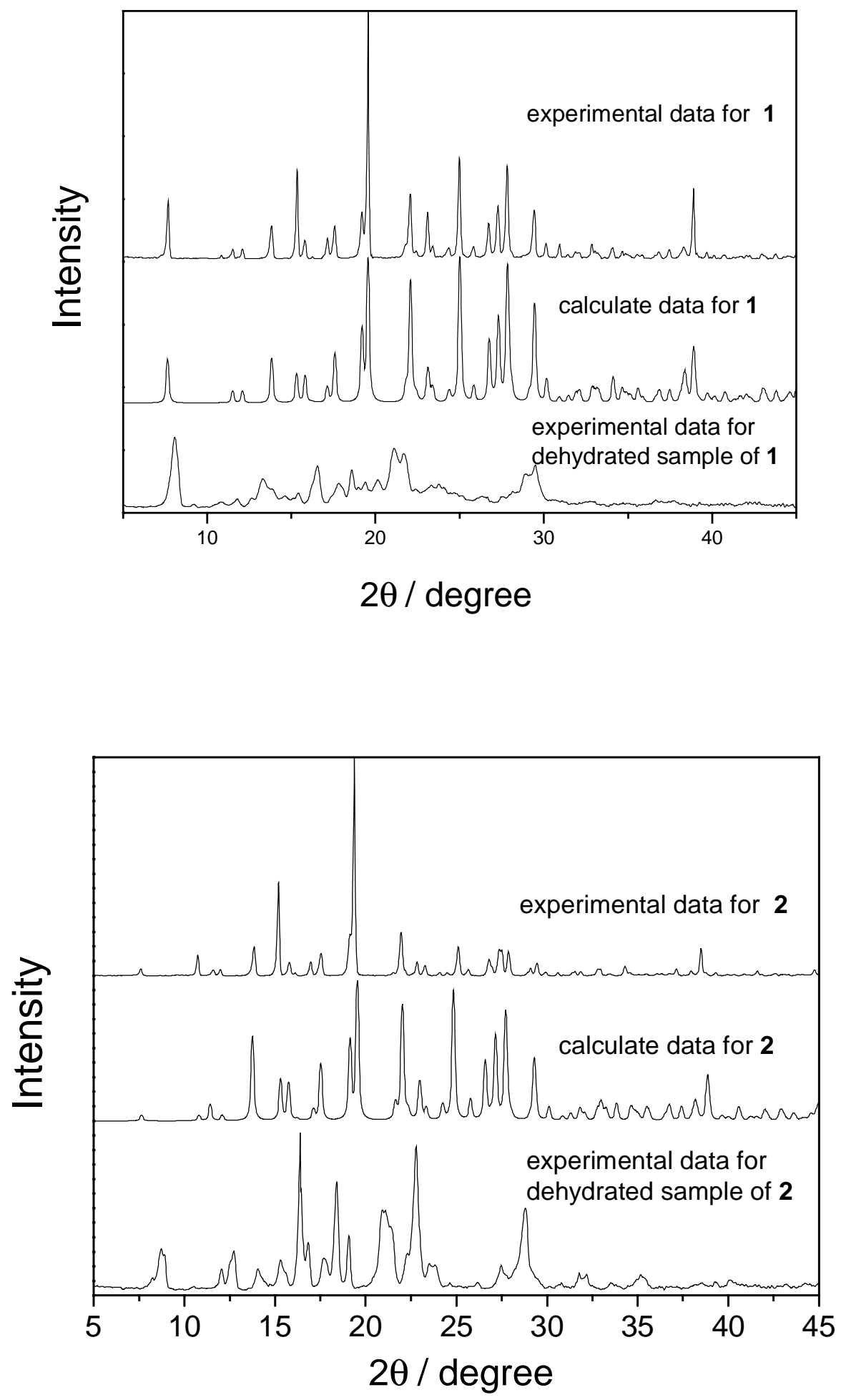

Fig. S2. XRPD patterns for compounds 1 and 2. 